\title{
Acceso abierto y su repercusión en la Sociedad del Conocimiento: Reflexiones de casos prácticos en Latinoamérica
}

\section{Open Access and its impact on the Knowledge Society: Latin American Case Studies Insights}

\author{
María Soledad Ramírez Montoya \\ Escuela de Educación, \\ Humanidades y Ciencias Sociales \\ Tecnológico de Monterrey \\ solramirez@itesm.mx \\ Fecha de recepción: 2-2-2015 \\ Fecha de revisión: 2-3-2015
}

\section{Palabras clave:}

Sociedad del conocimiento; acceso abierto; repositorios; revistas y políticas.

\section{Resumen}

En una sociedad cambiante, el acceso abierto puede representar una alternativa de crecimiento y recursos para la comunidad educativa, desde las posibilidades que da a los estudiantes, hasta los profesores, investigadores y gestores de instituciones educativas. El objetivo de este artículo es analizar las oportunidades y retos que da el acceso abierto para la comunidad educativa, a través de la presentación de una visión conceptual y de casos prácticos en América Latina, con respecto al tema de los recursos educativos abiertos, los repositorios, revistas y políticas de acceso abierto -de las universidades y del gobierno o agencias de financiación- y su vinculación con una sociedad del conocimiento. Las conclusiones se presentan en torno a tres elementos clave: posibilidades, retos y oportunidades en el acceso abierto para la sociedad del conocimiento.

\section{Fecha de aceptación: 20-3-2015 Fecha de publicación: 27-3-2015}

Keywords:

Knowledge society; open repositories; journals and open access policies.

In a changing society, open access may represent an alternative growth and resources to the educational community, from the opportunities given to students, to teachers, researchers and administrators of educational institutions. The aim of this paper is to analyze the opportunities and challenges that gives open access to the educational community, through the presentation of a conceptual vision and practical cases in Latin America, on the issue of open educational resources, repositories, journals and open access policies -from universities and government agencies or financingand its link to a knowledge society. The findings are presented on three key elements: opportunities, challenges and opportunities open to access the knowledge society. 


\section{Introducción}

El término "sociedad del conocimiento" se presenta aún difuso en la variedad de contextos internacionales. Si bien es cierto que la impronta del avance del Internet y las tecnologías de la información y la comunicación ha dado paso a nuevas formas de generación, distribución y movilización del conocimiento, no se debe olvidar que el "acceso" a estos avances y el uso mismo de la información no garantizan, automáticamente, mejoras en los ámbitos sociales, culturales y económicos. En la búsqueda de la concreción de una "sociedad del conocimiento", el acceso abierto en el ámbito educativo se presenta como una oportunidad para mejorar la transferencia de conocimiento para tratar de producir mejores bienes y servicios. El movimiento educativo abierto se conforma a partir de dos iniciativas: la difusión del trabajo científico en medios de acceso abierto y la movilización del conocimiento a través de la producción, distribución, uso y reúso de recursos educativos abiertos (REA) para incidir en prácticas educativas. Solo con base en el conocimiento, los países tendrán la oportunidad de humanizar el proceso de globalización (United Nations, Educational, Scientific and Cultural Organization [UNESCO], 2005). Desde esta perspectiva se puede detectar en varias partes del mundo que los gobiernos y las instituciones de educación superior han implementado proyectos que buscan diseminar el conocimiento a través de repositorios en los que se aloja la producción científica y educativa, permitiendo el acceso de forma libre y gratuita a un gran número de recursos educativos. Sin embargo, como resultado de diversos factores como la capacidad económica, el interés de las universidades y el conocimiento de los actores del ámbito educativo, entre otros, el avance en las diferentes regiones ha sido desigual. En América Latina, por ejemplo, el desarrollo de iniciativas en torno al movimiento de acceso abierto es aún incipiente (Babini, 2011; Galina y Giménez, 2008). Si bien, se puede considerar la publicación del trabajo intelectual de los cuerpos de investigadores y académicos del continente en revistas comerciales reconocidas a nivel mundial y se puede hablar de un avance en la presencia de la región (Albornoz, 2010), es importante también reconocer que la difusión de la producción científica en acceso abierto muestra un fuerte rezago. Enestemarco, elaccesoabiertose presentacomo una oportunidad para los países emergentes. En especial, Altbach (2013) menciona que las universidades de investigación en los países en desarrollo deben dar especial atención al trabajo intelectual de sus investigadores para que contribuyan con éxito a una sociedad basada en el conocimiento. Todos los países en desarrollo necesitan desarrollar este tipo de instituciones para participar en el entorno globalizado de la educación superior. Además de la atención al trabajo intelectual, se deben atender dos elementos sustanciales: la construcción de infraestructura necesaria para la investigación y el generar un medio ambiente intelectual, que ayuden a las instituciones en sus propósitos deinvestigación. El objetivo de este escrito es analizar las oportunidades que da el acceso abierto para la comunidad educativa, a través de la presentación de una visión general sobre el estado actual del acceso abierto en Latinoamérica, con respecto al tema de los recursos educativos abiertos, los repositorios, revistas y políticas de acceso abierto - de las universidades y del gobierno o agencias de financiación- y su vinculación con una sociedad del conocimiento. El escrito transcurre por apartados que van desde una visión conceptual de la sociedad del conocimiento, pasando por la presentación de experiencias mundiales, hasta latinoamericanas, y se cierra con unas conclusiones que enuncian las posibilidades, retos y oportunidades que se visualizan en este caminar del acceso abierto. 


\section{Marco conceptual: Tintes de una sociedad del conocimiento vinculada con el acceso abierto}

Las posibilidades de los avances tecnológicos se han relacionado con accesibilidad y competitividad. Drucker (2004) indicó que la sociedad del conocimiento será cada vez más competitiva, dado que el conocimiento es ahora universalmente accesible; también Tedesco (2000) menciona que la gestión del flujo de información crea posibilidades abiertas por las tecnologías digitales, que incluso han borrado las fronteras polí $\neg$ ticas y sociales en la comunicación y la información. La idea de una sociedad del conocimiento viene a reconocer la importancia fundamental que en todos los países tiene la generación de conocimiento y el desarrollo de tecnologías apropiadas a las realidades locales (Marrero, 2007). De tal forma que hablar de una sociedad del conocimiento es hablar de vincular el saber con la aplicación del conocimiento. Una sociedad del conocimiento visualiza la educación continua y el desarrollo de competencias para la resolución de problemas en una sociedad cambiante. García (2008) menciona que, en la sociedad del conocimiento, el aprendizaje no se circunscribe a un determinado espacio y se exige aprender en todos los contextos, sin limitarse a un periodo temporal en el ciclo vital de la persona. En la sociedad del conocimiento, se postula por la asimilación de una base de conocimientos rigurosos y estrategias eficaces y por desarrollar un pensamiento reflexivo, crítico y creativo. Los cambios sociales, en este tipo de sociedad, promueven transformaciones también en la educación. De Pablos (2010) hace hincapié en que este nuevo marco que configura la sociedad de conoci $\neg$ miento plantea nuevas exigencias a los sistemas educativos $\mathrm{y}$, por tanto, también a la universidad, ya que cada vez la educación debe responder con más calidad a las demandas sociales. Carneiro (2010) alerta además en que las infraestructuras y redes del saber surgen como el motor de un nuevo diseño de sociedad: la sociedad del conocimiento. Las instituciones educativas deben cambiar en la misma medida que lo hacen las sociedades en las que se asientan. Se puede deducir, a través de lo planteado, que una sociedad del conocimiento postula por usar el saber a través de diversos mecanismos que pasan por las personas, los cambios y los fines. En esta visión, lkka (2013) plantea diferentes modelos de implementación de las cuatro funciones sociales de la educación en este tipo de sociedad del conocimiento: (1) red de información, (2) el aprendizaje continuo, significado, procesamiento y creación de conocimiento, (3) la diversidad cultural con trabajo en comunidades y redes y (4) la realización de las capacidades humanas, aumentar la capacidad de dar cuenta del valor y tomar decisiones. Con estos mecanismos se delinean algunas posibilidades para abrir el conocimiento. En la configuración de este tipo de sociedades, el acceso abierto puede coadyuvar a hacer accesible el saber. El acceso abierto a la literatura significa su disponibilidad gratuita en Internet, permitiendo a cualquier usuario leer, descargar, copiar, distribuir, imprimir, buscar o enlazar los textos completos de estos artículos. La única restricción es sobre la reproducción y distribución, y el tipo de licenciamiento que den los autores que les permita asegurar la integridad de su trabajo y el derecho a ser debidamente reconocido y citado (Iniciativa de Acceso BudapestOpen, 2002). El término educación abierta se refiere a las formas de la educación en las que las ideas o aspectos importantes de la metodología de la enseñanza o de la infraestructura se comparten libremente en Internet (Coerll, 2013). El conocimiento abierto es presentado por García-Peñalvo, García de Figuerola y Merlo (2010) como la suma de cuatro áreas: software, contenido, ciencia e innovación. Acceso abierto se vincula, de esta forma, a la gratuidad del saber, con reconocimiento a sus autores. Son múltiples los argumentos a favor del acceso abierto. La European Commission 
(2013) informa razones de alto nivel basados en principio de un argumento moral, que es que los resultados de la investigación financiada con fondos públicos deben ser accesibles al público y que se debe permitir que los resultados dela investigación sean compartidos con el público en general, ayudando a crear una sociedad del conocimiento, así como el que debe apoyarse para la transferencia de conocimiento a los sectores que pueden utilizar directamente ese conocimiento para producir mejores bienes y servicios. Sin embargo, los problemas no se eximen en el ámbito del acceso abierto. Aliaga (2012) menciona que las grandes editoriales se han dado cuenta de que el movimiento de Open Access, gracias a las herramientas disponibles, permiten que los propios investigadores desarrollen sus propios medios de comunicación, con lo que buena parte del negocio, basado en la intermediación (la oferta de contenido proviene de los investigadores y la demanda también) podría entrar en crisis y el número de suscripciones pudiera llegar a reducirse. Nicolosi y Ruivenkamp (2013) también mencionan las dificultades para los científicos, que a menudo no tienen la libertad de elegir la forma de organizar los temas de patentes porque los derechos de patente, de manera diferente a los derechos de autor sobre los textos, están en manos de sus instituciones empleadoras (universidades, organismos de investigación, etc.). En la comunidad académica los retos del acceso abierto para la sociedad del conocimiento son múltiples. Lourenço y Borrell-Damian (2014) destacan varios desafíos que enfrentan las universidades, por ejemplo, la necesidad de sensibilización y promoción de acceso abierto dentro de la comunidad académica y la adopción de modelos de negocio rentables, compatibles con las limitaciones del presupuesto universitario. Cox (2013) también menciona que hay una tensión en torno a las reglas explícitas de promoción de la institución, enuncia que existen dudas acerca de la naturaleza de la comunidad que va a utilizar sus materiales de enseñanza y si los materiales podrían ser utilizados en su forma actual en diferentes contextos $y$, finalmente, cita el que existen dudas acerca de la calidad y el tipo de materiales que serían compartidos, y si los materiales son adecuados. En contraposición, el avance y los resultados explícitos del acceso abierto han otorgado evidencias de su impacto. Gunasekaran y Arunachalam (2014), por ejemplo, midieron el impacto de las revistas de acceso abierto y de suscripción. Su estudio muestra que muchas revistas de países en desarrollo, especialmente de América Latina y la India han mejorado su visibilidad e impacto mediante la adopción de acceso abierto. Melero y Hernández-SanMiguel (2014) mencionan que la cohesión de tres pilares: contenido, procesos e infraestructura de componentes "abiertos" ("opens") conduce a un cambio cultural. Pero si se produce la desfragmentación en los "opens" puede conducir a la toma de decisiones buenas para unos, que podrían ser adversas para otros, además la sostenibilidad sería más arriesgada y el aprovechamiento de los recursos no sería óptimo. Para alcanzar este "estado de abiertos" los autores hacen las siguientes recomendaciones:

- Que las instituciones financiadoras, académicas y de investigación adopten políticas basadas en el abierto como modus operandi para cualquier actividad financiada con fondos públicos.

- Estaspolíticasdebenincluir procedimientos para el seguimiento de su cumplimiento.

- La colaboración e implicación de los investigadores debe incentivarse por:

$\begin{array}{llr}\text { - Adopción } & \text { de } & \text { nuevos } \\ \text { sistemas } & \text { apropiados } & \text { de }\end{array}$

- Servicios de apoyo con respecto a los derechos de autor y licencias.

- Capacitación dirigida al personal de la institución.

- Asegurarse que la interoperabilidad de los sistemas y servicios sea un componente principal de la e-infraestructura abierta. Con base en estos tintes conceptuales de una sociedad del conocimiento vinculada con el acceso abierto, se plantea a continuación ¿cómo se manifiestan estas ideas en casos prácticos de la realidad? 


\section{Casos prácticos: de las experiencias mundiales de acceso abierto a las experiencias latinoamericanas}

En el ámbito internacional han ido apareciendo iniciativas muy interesantes de acceso abierto, según la Organización para la Colaboración y el Desarrollo Económico ([OCDE], 2007) aunque no es posible dar una estimación exacta del número de iniciativas, algunos ejemplos son:

- OpenCourse Ware (OCW, por sus siglas en inglés) del Instituto Tecnológico de Massachusetts (http://www. ocwconsortium.org/). En su etapa inicial este proyecto contó con fondos financieros de la Fundación Hewlett y la Fundación Mellon.

- MERLOT (por sus siglas en inglés) (http://www.merlot.org/merlot/index. htm). Se ofrecen recursos educativos producidos por la Universidad de California State

- OpenLearn de la Universidad Abierta del Reino Unido (http://openlearn. open.ac.uk/). Este portal en línea cuenta con dos modalidades: consulta de materiales didácticos en el LearningSpace y el LabSpace donde el material puede transmitirse, adaptarse y reutilizarse.

- MORIL (por sus siglas en inglés) (http:// moril.eadtu.eu/). Integra recursos de las universidades más importantes de nueve países de Europa. Los materiales son producidos en diferentes idiomas.

- Universidad de Western Cape en Sudáfrica (http://opencontent.uct.ac.za/). Esta universidad ha puesto en marcha una iniciativa para ofrecer recursos digitales y software de manera libre y gratuita.

- OpenER de la Universidad de los Países Bajos (http://dspace.ou.nl/). En el portal de esta universidad se dispone de 400 horas de cursos en holandés, dirigidos para el público en general interesado en el auto-aprendizaje.

Otros ejemplos de proyectos desarrollados en el contexto europeo, son ARIADNE (por sus siglas en inglés) (http://www.ariadneeu.org/), PROMETEUS (por sus siglas en inglés) y el Repositorio de Buenas Prácticas de Innovación Educativa. El objetivo de ARIADNE es conectar diversos repositorios, para poner a la disposición recursos digitales de alta calidad. PROMETEUS es una iniciativa fundada por la Unión Europea, cuyo propósito es facilitar el acceso al conocimiento y la educación a los ciudadanos de Europa, sin importar el país o el idioma (Alves y Uhomoibhi, 2010). El Repositorio de Buenas Prácticas de Innovación Educativa (http://138.4.83.162/organiza/buscador buenaspracticas/) está dirigido a profesores y se centra en la aplicabilidad de experiencias innovadoras (García-Peñalvo, 2015, citando a Fidalgo, 2012).

En Portugal existe una iniciativa muy interesante, el RCAAP (www.rcaap.pt) que integra el Repositorio Científico de Acceso Abierto de Portugal. El RCAAP reúne e integra los contenidos de los repositorios institucionales portugueses y constituye un único punto de entrada para la búsqueda, el descubrimiento y la recuperación de miles de documentos científicos y publicaciones académicas, a saber, los artículos de revistas, ponencias en congresos, tesis y disertaciones, distribuidos por varios repositorios. Este proyecto fue desarrollado por la FCCN Fundação para a Computação Científica Nacional, con la colaboración técnica y científica de la Universidad de Minho.

Estos son algunos ejemplos de iniciativas promovidas alrededor de todo el mundo en torno a la diseminación del conocimiento, a través de sitios en línea. En algunos casos, estas iniciativas se generan dentro de las instituciones educativas y en otros, los proyectos surgen de organizaciones interesadas en ampliar las posibilidades de acceso a la educación, a través de materiales digitales cuya calidad esté garantizada.

América Latina también ha promovido algunos proyectos, como la Red Latinoamericana de Portales Educativos (RELPE) (http:// www.relpe.org/). Surgida a partir de la 
iniciativa de los presidentes de algunos países del continente, actualmente RELPE está conformada con 24 miembros. El objetivo de la Red es compartir recursos educativos y software libre entre otras herramientas, a fin de apoyar la tarea de los profesionales de la educación (RELPE, 2011).

$\mathrm{Si}$ se compara con las universidades de Estados Unidos y algunas de Europa o Asia, la participación de las instituciones de educación superior latinoamericanas en el movimiento educativo abierto es aún limitada. Destaca, sin embargo, el esfuerzo de universidades brasileñas y mexicanas para incrementar el número de portales en los cuales se comparten recursos educativos (Aguado, Rogel, Becerril y Baca, 2009). Otro ejemplo es la iniciativa aprobada por la Universidad de la República en Uruguay en 2008, en la que se establece el compromiso para publicar en formato abierto toda la producción científica de esa universidad (Universidad de la República, 2008). La Universidad Nacional del Plata en Argentina también ha implementado políticas institucionales para promover la publicación de la producción intelectual de sus investigadores, académicos y estudiantes en su portal educativo (Universidad Nacional de La Plata, 2012).
Con la experiencia de trabajo en comunidades de aprendizaje virtuales, en 2011 se creó la Comunidad Latinoamericana Regional de Investigación Educativa y Social (CLARISE, portal Web https://sites.google.com/site/ redclarise/ (Figura 1). Surgida a partir de una convocatoria de RedCLARA, en su programa COMCLARA 2011, el Tecnológico de Monterrey, junto con universidades de cinco países del continente, conformaron CLARISE. Entre las instituciones participantes se encuentran las universidades de Palermo, Nacional de Córdoba, Nacional de San Juan y Nacional del Mar del Plata en Argentina; las universidades de La Sabana y de Santo Tomás (Bucaramanga) en Colombia; la Universidad de Costa Rica y Cisco Systems en Costa Rica y la Universidad de la República en Uruguay. CLARISE ha permitido el intercambio de experiencias y conocimientos entre investigadores, académicos y estudiantes de las instituciones educativas participantes (Ramírez y Burgos, 2012). Además, se ha logrado la publicación de artículos y ponencias que se encuentran disponibles de manera libre y gratuita en el portal de la comunidad, permitiendo así tener mayor visibilidad de los trabajos realizados (Ramírez, 2012).

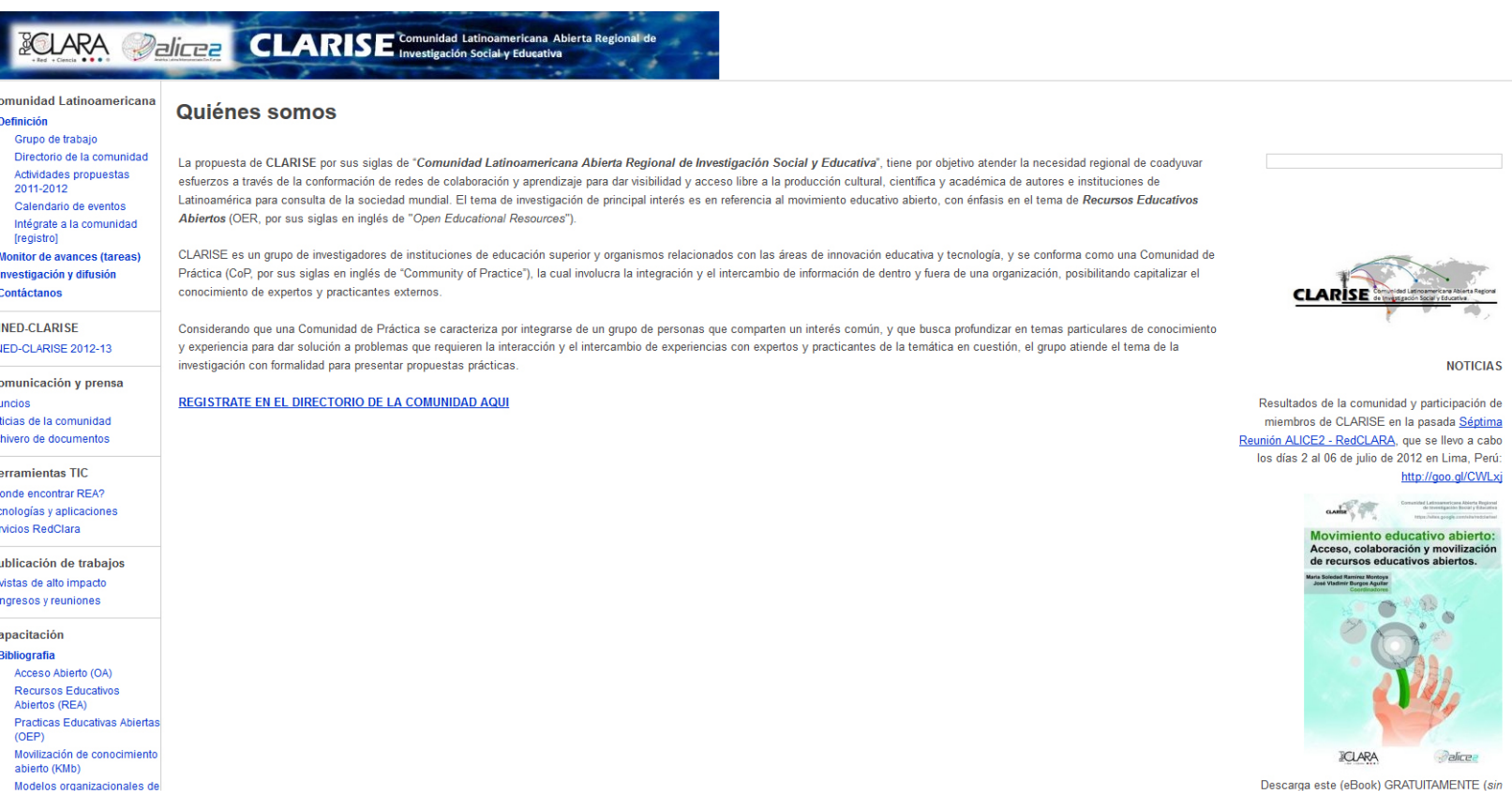


Otro proyecto impulsado por la RedCLARA con financiamiento del Banco Interamericano de Desarrollo (BID) en torno al movimiento educativo abierto en el contexto regional, es la Red Federada de Repositorios Institucionales de Publicaciones Científicas, denominado LAReferencia. Esta iniciativa tiene como objetivo dar visibilidad a la producción científica generada en las instituciones de educación y de investigación (Red Federada de Repositorios Institucionales de Publicaciones Científicas-LAReferencia, 2012). En el proyecto, actualmente participan ocho países de la región, entre los que se encuentran Argentina, Brasil, Chile, Colombia, Ecuador, México, Perú y Venezuela y se espera establecer una estrategia regional consensuada para la interconexión de repositorios, un marco común de acuerdos y políticas para la interoperabilidad, un modelo operativo con base en el proyecto piloto, así como planes de acción para capacitación en el contexto regional (Rodríguez y Lavariega, 2011).

En México, las universidades han comprendido que en esta nueva sociedad globalizada, la compartición del conocimiento es condición necesaria para un desarrollo más equilibrado de las naciones. Algunos de los ejemplos más conocidos son la Universidad Nacional Autónoma de México (UNAM), que cuenta con diversos acervos digitales como el índice de revistas IRESIE (Índice de Revistas de Educación Superior e Investigación Educativa) y el repositorio PodcastUNAM, así como la participación en proyectos internacionales como Latindex y la Scientific Electronic Library on Line (SciELO) (Galina y Giménez, 2008). Además, a partir de 2012, la UNAM, a través de su Sistema de Universidad Abierta y Educación a Distancia (SUAyED), ofrece cerca de 3.000 materiales digitales diseñados por académicos de esa casa de estudios, que abarcan las diversas áreas del conocimiento y pueden ser usados en los diferentes niveles educativos: desde secundaria hasta posgrado (UNAM-SUAyED, 2012).

La Universidad Autónoma del Estado de México (UAMEX) desarrolló el Sistema de Información Científica REDALYC, en el cual actualmente se alojan 225.818 artículos de 758 revistas científicas, publicadas en 19 países de América Latina, el Caribe y
Europa (REDALYC, 2012). En REDALYC, los investigadores y académicos de la región tienen a su disposición un gran acervo de materiales que pueden ser consultados de forma libre y gratuita. Por su parte, con el propósito de fortalecer el proceso de enseñanza-aprendizaje y contribuir en la formación integral de estudiantes de nivel medio-superior y superior, la Universidad de Guadalajara ha desarrollado el repositorio de recursos educativos CREA -Centro de Recursos para la Enseñanza y el Aprendizaje-, (Universidad de Guadalajara-CREA, 2012).

Según el registro del Directory of Open Access Repositories (DOAR, por sus siglas en inglés) desarrollado por el Centre for Research Communications de la Universidad de Nottingham, en México existen 20 repositorios digitales de acceso abierto (DOAR, 2012). Además de los ejemplos señalados anteriormente, otras instituciones y universidades que cuentan con repositorios son el Colegio de Posgraduados, el Instituto Nacional de Salud Pública, el Instituto Politécnico Nacional (IPN), el Instituto Tecnológico de Estudios Superiores de Occidente (ITESO), la Universidad Autónoma de Nuevo León (UANL), la Universidad Autónoma del Estado de Hidalgo (UAEH), Universidad de las Américas Puebla (UDLAP), Universidad del Claustro de Sor Juana y Universidad Veracruzana. En dichos portales se alojan materiales como tesis, artículos y objetos de aprendizaje.

El Tecnológico de Monterrey también se ha destacado por impulsar diversos proyectos relacionados con la diseminación de la producción científica de su comunidad. El primer paso en ese camino fue la adopción y transferencia de los cursos ofrecidos por instituciones educativas de Estados Unidos como Carnegie Mellon University (OLI, por sus siglas en inglés), Yale University (OYC, por sus siglas en inglés) y el Instituto Tecnológico de Massachusetts (MIT-OCW, por sus siglas en inglés) (Burgos y Ramírez, 2010).

A partir de la experiencia adquirida, en enero de 2007, en el marco del Foro Económico Mundial en Davos, Suiza, se presentó la primera iniciativa generada dentro del Tecnológico de Monterrey: el portal 
Knowledge Hub (KHub, hoy llamado Temoa http://www.temoa.info). Este proyecto fue nombrado como uno de los casos de éxito de
América Latina por el Banco Interamericano de Desarrollo en el 2014 (Figura 2).

\section{temoa

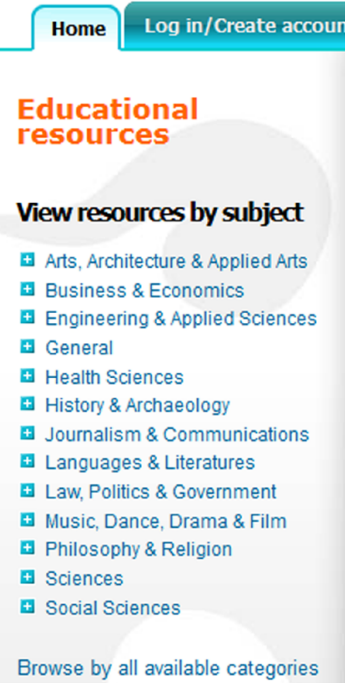

Open Educational Resources Portal

Tecnológico de Monterrey

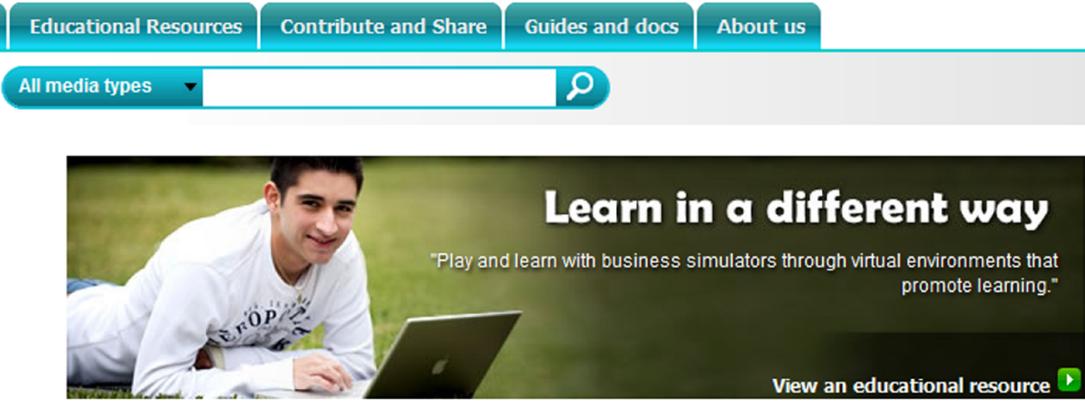

In temoa, you will find:

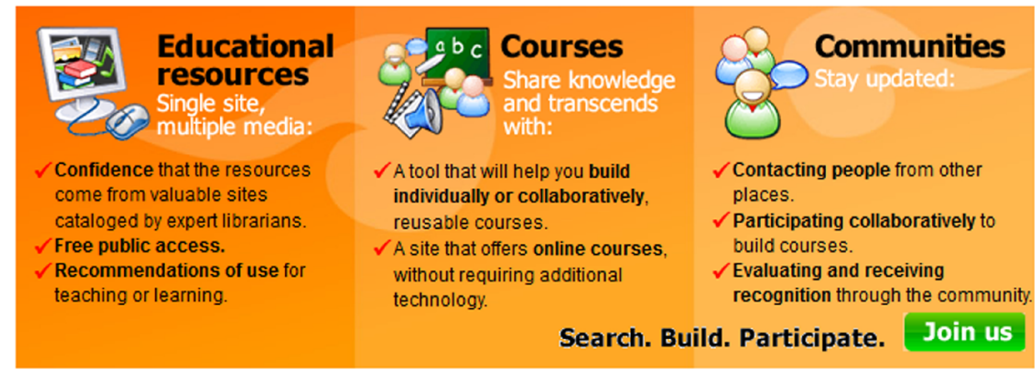

Popular resources

- Reviews: enhanced functionality to share educational experiences KHUB in the "School of the Future World Summit 2008"

- KHUB in Dubai: World Economic Forum: Summit on the Global Agenda 2008 هRecent News See more

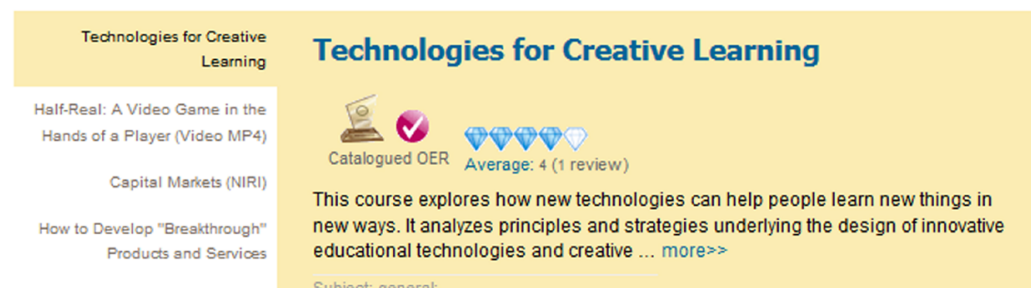

Figura 2. Temoa-Portal de Recursos Educativo Abiertos (http://www.temoa.info/)
En 2010 se trabajó en la formación de investigadores en el uso y producción de recursos educativos abiertos, que actualmente se encuentran alojados en el repositorio DAR (Desarrolla, Aprende y Reutiliza) (http://catedra.ruv.itesm.mx/) (Ramírez y Burgos, 2012). Finalmente, como resultado del proyecto 2011, se desarrolló un metaconector de repositorios denominado Educonector.info y se editó una guía de uso de recursos educativos abiertos, que conjunta la experiencia de investigadores y docentes (Mortera y Ramírez, 2013). Cabe señalar que estos proyectos abrieron la oportunidad de trabajar en comunidades de práctica virtuales, con investigadores y académicos de diversas universidades e institutos de educación superior en México y Latinoamérica, tanto de investigación, como de formación a través de cursos masivos abiertos (MOOC, por sus siglas en inglés). En la Figura 3 se muestran algunos ejemplos de MOOC impartidos por CLARISE y el Tecnológico de Monterrey.

En el marco de este proyecto, en México se conformó la Red Mexicana de Repositorios Institucionales (REMERI), que cuenta con el apoyo de la Corporación Universitaria para Internet (CUDI) (Rodríguez, 2011). Entre los objetivos de REMERI, se pretende crear un nodo que permita la interconexión de repositorios institucionales. En la primera etapa se pretende conectar los repositorios que ya cuentan con las condiciones de interoperabilidad necesitarías, así como apoyar y dar capacitación a las instituciones educativas que aún no cuentan con 


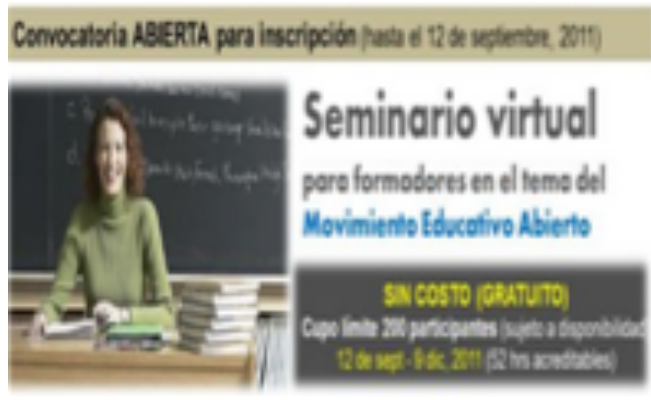

\section{MOOC: Teaching skills \& OER (2013)}

\section{MOOCs: Innovation \& C $\left(2013^{*}\right.$ and $\left.2014^{* *}\right)$}
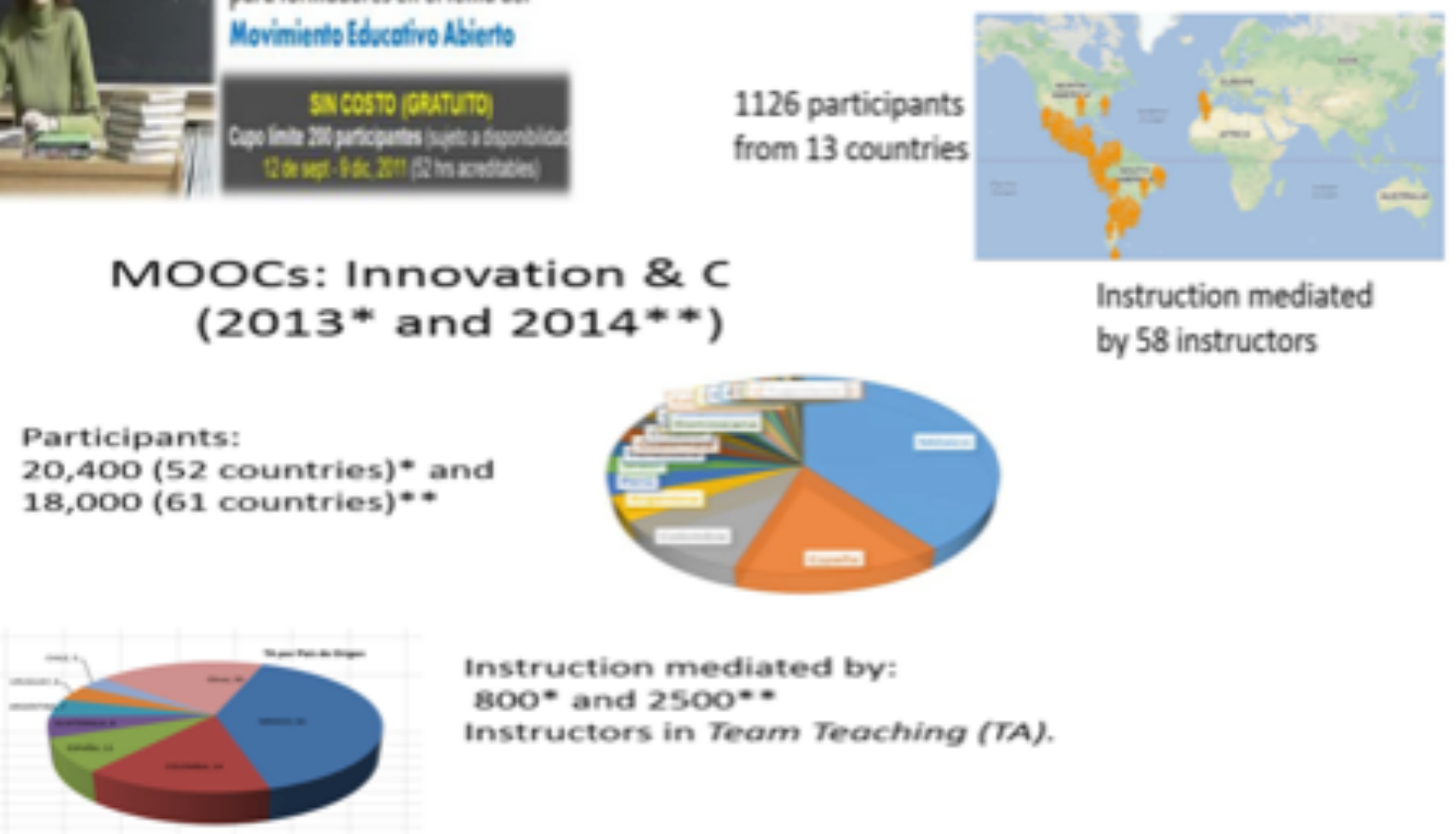

Instruction mediated by:

$800^{*}$ and $2500^{* *}$

Instructors in Team Teaching (TA).

\section{Figura 3. MOOC impartidos por CLARISE y Tecnológico de Monterrey}

repositorios. En el proyecto actualmente participan seis instituciones de educación superior: la Universidad Autónoma de San Luis Potosí (UASLP), el Tecnológico de Monterrey (ITESM), la Universidad de las Américas Puebla (UDLAP), la Universidad Autónoma del Estado de Hidalgo (UAEH) y la Universidad de Guadalajara (UdG) (Vázquez, 2011).

La iniciativa de ley en materia de acceso abierto fue aprobada en México en mayo del 2014 dicha iniciativa propone concretamente la reforma las leyes generales de Ciencia y Tecnología y de Educación con el fin de difundir las publicaciones científicas de forma libre (http://www.dof.gob.mx/nota_detalle. php? codigo $=5345503 \&$ fecha $=20 / 05 / 2014)$. La iniciativa citada surge en un contexto de cambios a nivel nacional relacionados con temas de gran relevancia como son: la reforma educativa, la reforma en telecomunicaciones y la reforma energética.

En forma sintética se presentan las iniciativas y acciones que conforman el estado actual del Movimiento Educativo Abierto que ha tenido lugar en México de acuerdo con el Portal
Global de Acceso Abierto de la UNESCO (2011):

- El Consejo Nacional de Ciencia y Tecnología (Consejo Nacional de Ciencia y Tecnología -CONACYT-) de manera conjunta con CUDI (Corporación Universitaria para el Desarrollo de Internet), así como otras organizaciones y universidades de México, conformaron el llamado Consorcio Nacional de Científicos y Tecnológicos de Información de Recursos de Información Científica y Tecnológica, que contribuye a mejorar la calidad del acceso y abierto de las revistas de revisión por pares de México.

- México cuenta actualmente con 97 revistas de acceso abierto en DOAJ y Latindex, ofreciendo enlaces a 73 publicaciones de revistas de acceso abierto de México.

- A través del directorio de repositorios de acceso abierto llamado OpenDOAR se identificaron más de 20 repositorios de universidades $y$ otras instituciones de investigación y enseñanza, sobre todo relacionados con tesis, artículos de revistas, objetos de aprendizaje, documentos de 
conferencias y multimedia.

- La Red Abierta de Bibliotecas Digitales (RABID) desarrolla actualmente un proyecto en conjunto con una red mexicana de repositorios institucionales llamada Red Federada de Repositorios Institucionales de RABID con el fin de trabajar en conjunto con CUDI y CONACYT, que son a la vez los puntos focales nacionales de RedCLARA en México, y los coordinadores nacionales de la Red Federada Latinoamericana de Repositorios institucionales de Documentación Científica.

- México también cuenta con miembros en CoLaBoRa, la Comunidad Latinoamericana de Bibliotecas y Repositorios Digitales.

- La Universidad Nacional Autónoma de México (UNAM) ha tenido un papel muy importante en el desarrollo de programas para dar visibilidad y el acceso abierto a las revistas de América Latina y el Caribe como LATINDEX, CLASE, PERIODICA, y ahora con su reciente incorporación a Scielo. México cuenta ya con la posibilidad de consultar 76 revistas de acceso abierto. Ejemplos: UNAM revistas electrónicas OJS portal (con acceso abierto a 53 revistas), RAD -UNAM- (cuenta con 6 repositorios institucionales de UNAM), REPOSITAL (recursos educativos abiertos UNAM), Humanindex (ciencias sociales y humanidades), entre otros.

- La Universidad Autónoma del Estado de México (UAEM) desarrolló el sistema de información científica Redalyc (http:// www.redalyc.org), con el objetivo de contribuir a la visibilidad y la accesibilidad de las revistas científicas de Iberoamérica. Hoy en día ofrece acceso libre y gratuito a artículos de texto completo de más de 640 revistas de revisión por pares de 13 países de América Latina y el Caribe. Incluye 169 revistas a texto completo de México.

- En México 201 revistas utilizan OJS software de código abierto y facilitan el acceso abierto a los artículos a texto completo.

- En elárea de la salud se cuenta con Artemisa en Línea (http://www.artemisaenlinea. org.mx), con 57 publicaciones de texto completo de revistas de salud mexicanas.

- La Universidad de Colima, con apoyo de la Oficina del Caribe de la Unesco, construyó el Portal INFOLAC (http:// infolac.ucol.mx) con el fin de generar y compartir conocimientos entre los bibliotecarios, documentalistas y gestores de información de la región para contribuir a la construcción de la Sociedad de la Información en América Latina y el Caribe.

- El Tecnológico de Monterrey cuenta con un sistema de indexación que proporciona acceso a objetos de aprendizaje de contenido abierto (http://www.temoa. info).

- La Universidad de las Américas de Puebla cuenta con acceso a publicación en la biblioteca digital de Tesis y Disertaciones (NDLTD) http://www.ndltd.org/search_ form

- México participa también en repositorios temáticos de acceso libre, en la actualidad con un número creciente de registros con textos completos, ejemplos: salud (BVS), la agricultura (SIDALC), la ciencia (PERIÓDICA), la educación (RELPE), la gestión pública y políticas (CLADSIARE), Ciencias Sociales (CLACSO, FLACSO, CLASE), ciencias del mar (OceanDocs), el trabajo (LABORDOC), ciencias de la información (E -Lis), entre otros.

Para concluir se hace mención a una unidad de inteligencia competitiva del Tecnológico de Monterrey dedicada al análisis y difusión de las tendencias educativas que están moldeando la educación del futuro (Figura 4), que tiene por objetivo el promover e impulsar el desarrollo de innovaciones educativas a través de la difusión de las tendencias y experiencias de mayor impacto en la educación superior, como una ventana de acceso abierto. También se enuncia el nombramiento de dos oficinas internacionales: en abril 2014 el International Council for Open and Distance Education nombra la oficina internacional de Recursos Educativos Abiertos para América Latina y en mayo de 2014 la UNESCO aprueba la Cátedra Movimiento Educativo Abierto para América Latina. Ambas oficinas internacionales a cargo del Tecnológico de 
Monterrey, para promover la formación, investigación y diseminación del tema en la comunidad Latinoamericana.

Con base en los casos prácticos presentados a nivel mundial, con especial énfasis en las experiencias latinoamericanas, se plantea a continuación ¿qué análisis se pueden realizar sobre el tema de acceso abierto y su repercusión en la sociedad del conocimiento?

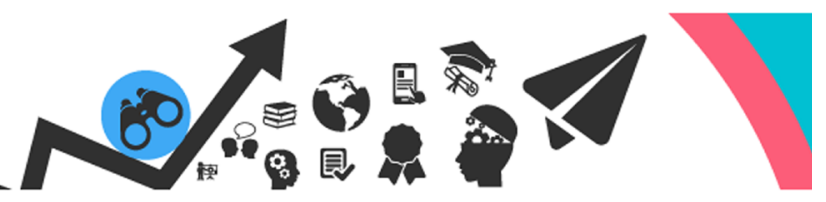

y $\mathrm{g}^{+}$in

IDENTIFICAMOS Y ANALIZAMOS LAS TENDENCIAS EDUCATIVAS QUE ESTÁN MOLDEANDO EL APRENDIZAJE DEL FUTURO
Q search

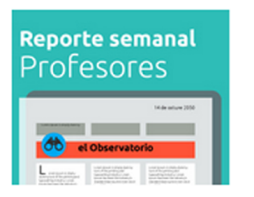

Reporte semanal profesores

Síntesis de medios con las notas y artículos
más relevantes en educación, tecnología e innovación para docentes.

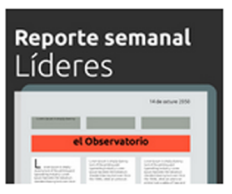

Reporte semanal líderes

Síntesis de medios con las notas y artículos más relevantes en educación, tecnología innovación para directivos académicos.

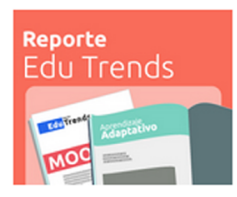

Reporte Edu Trends

Análisis de mayor profundidad sobre aquellas tendencias con mayor potencial de impacto en la

educación superior.
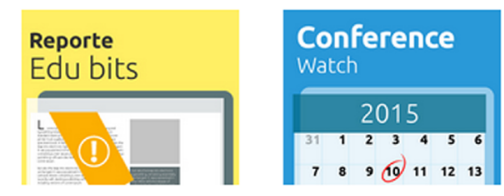

Reporte

Edu bits

Análisis condensados de temas estratégicos para la educación.
Conference Watch

Agenda de los eventos

más relevantes en el

mundo sobre

innovación educativa.

\section{Algunas reflexiones en el camino del acceso abierto y su repercusión en la sociedad del conocimiento}

En el área de acceso abierto, se puede encontrar un extenso número de organizaciones e instituciones educativas en todo el mundo que han desarrollado interesantes proyectos y en este ámbito, algunas de las universidades de América Latina han desarrollado iniciativas cuyo fin es la generación y compartición del conocimiento generado por los investigadores, académicos y estudiantes de la región. Sin embargo, de forma similar a los cuestionamientos a nivel internacional, la investigación sobre el uso que la comunidad latinoamericana hace del conocimiento generado sea aún incipiente (en contraposición de lo que Altbach, 2013, postula como requisitos para impulsar a las universidades de investigación, en una sociedad basada en el conocimiento), de ahí que en el cierre de este artículo visualizaremos posibilidades, retos y oportunidades.
Las posibilidades para que el contexto latinoamericano camine hacia una sociedad basada en conocimiento con apoyo del acceso abierto son múltiples. lkka (2013) plantea diferentes modelos de implementación en este tipo de sociedad del conocimiento (redes, aprendizaje continuo, comunidades y desarrollo de capacidades), también GarcíaPeñalvo, García de Figuerola y Merlo (2010) nos presentan la suma de cuatro áreas en el contenido abierto (software, contenido, ciencia e innovación) y, en ese sentido, en el estudio y análisis de cuestiones como el desarrollo de buenas prácticas de repositorios, la adopción de recursos educativos abiertos en la práctica educativa, la participación de los investigadores, académicos y estudiantes en comunidades virtuales, la transformación en los modos de pensar y hacer de las comunidades de profesionales de la educación, 
constituyen un interesante y valioso campo para desarrollos e investigación.

El reto viene al enfrentar múltiples barreras para el uso de recursos educativos abiertos en las prácticas educativas; se ha encontrado que el idioma es un límite importante, ya que el $72 \%$ de los recursos disponibles están en inglés (DOAR, 2012), lo que dificulta su uso en países que no son angloparlantes; la carencia de habilidades para el manejo de la tecnología; la desconfianza o resistencia de los productores y usuarios para usar recursos desarrollados por otras personas y la falta de políticas institucionales claras con respecto al respeto a los derechos de autor. Estos retos coinciden con lo enunciado por Cox (2013) con respecto al desconocimiento y desconfianza de la calidad de los REA, de ahí que para revertir este reto se hace necesario trabajar en la formación de la comunidad académica en su conjunto.

Las posibilidades están en visualizar de qué forma el Movimiento Educativo Abierto puede presentarse como un fenómeno dinámico, en constante definición y/o redefinición. Desde la idea más simple, pero tampoco sencilla, que consiste en aprovechar el potencial que ofrecen las Tecnologías de la Información y la Comunicación (TIC) para compartir un bien común: el conocimiento, hasta la más compleja que significa llevarla a las prácticas educativas, con el fin de que aporte a las mejoras requeridas de los procesos formativos, de gestión y de investigación. Melero y Hernández-San-Miguel (2014) postulan por la cohesión de los tres pilares que van desde el contenido, los procesos y la infraestructura de componentes "abiertos" ("opens") para conducir a un cambio cultural y, con ello, el Movimiento Educativo Abierto en Latinoamérica tiene una buena oportunidad para avanzar hacia una sociedad del conocimiento compartido.

El camino recorrido por otras instituciones e investigadores constituye un referente a seguir, en especial por el espíritu de colaboración que ha tenido su devenir en el área de acceso abierto. La sociedad del conocimiento requiere generar nuevos ambientes, nuevas relaciones entre los sujetos y necesita también un soporte estructural para gestionar estos entornos cambiantes. Queda con este escrito una posibilidad de crecimiento para seguir contribuyendo hacia la concreción de una sociedad basada en el conocimiento que nos permita superar los retos y aprovechar las potencialidades que el acceso abierto pudiera otorgarnos.

\section{Referencias}

Aguado, E., Rogel, R., Becerril, A. \& Baca, G. (2009). Presencia de universidades en la Red: La brecha digital entre Estados Unidos y el resto del mundo. Revista de Universidad y Sociedad del Conocimiento, 6(1), 1-17. Recuperado de

http://rusc.uoc.edu

Altbach, P. G. (2013). Advancing the national and global knowledge economy: the role of research universities in developing countries. Studies in higher education, 38(3), 316-330, http://dx.doi.org/10.1080/03075079.2013.7 73222
Albornoz, M. (2010). El estado de la ciencia en imágenes. En El estado de la ciencia. Principales indicadores de ciencia y tecnología Iberoamericanos / Interamericanos. Red Iberoamericana de Indicadores de Ciencia y Tecnología (RICYT). Buenos Aires, Argentina. Recuperado de: http://www.oei.es/salactsi/ESTADO2010. pdf

Aliaga, F. M. (2014). Veinte años de publicación electrónica y de acceso abierto: la madurez de una pionera. Relieve, 20(1). http://dx.doi.org/10.7203/relieve.20.1.3856 
Alves, P. \& Uhomoibhi, J. (2010). Issues of e-learning standards and identity management for mobility and collaboration in higher education. Campus-Wide Information Systems, 27(2), 79-90.

http://dx.doi.org/10.7203/relieve.20.1.3856

Babini, D. (2011). Acceso abierto a la producción científica de América Latina y el Caribe. Identificación de principales instituciones para estrategias de integración regional. Revista Iberoamericana de Ciencia, Tecnología y Sociedad CTS, 17(6), 31-56. Recuperado en:

http://revistacts.net/files/Volumen $\% 206 \% 20$ \%20N\%C3\%BAmero\%2017/Babini.pdf

Carneiro, R. (2007). La "nueva educación" en la sociedad de la información y de los saberes. Presentado en la sección Sociedad de la información y cambio educativo de la XXII Semana Monográfica Santillana de la Educación. Las tecnologías de la información y la comunicación (TIC) en la educación: retos y posibilidades. Disponible en:

http://www.temoa.info/go/49673

BudapestOpen Access Initiative (2002). Disponible en

http://www.budapestopenaccessinitiative. org/read

Burgos, J. V. \& Ramírez, M. S. (2010).Open Educational Resources: Experiences of use in a Latin-American context. Ed Open ED2010 Proceedings. Barcelona, España: UOC, OU, BYU. Disponible en

http://hdl.handle.net/10609/5062

Burgos, J. V. \& Ramírez, M. S. (2013). Academic knowledge mobilization to promote cultural change towards openness in education. En McGreal, R. Kinuthia, W., Marshall, S. y McNamara, T. (Editors.), Perspectives on open and distance learning: open educational resources: innovation, research and practice [pp.17-32]. Canadá: Commonwealth of Learning y la Cátedra de la UNESCO en la Universidad de Athabasca (UNESCO/COL CHAIR IN OER).
Coerll, C. B. (2013). Special issue commentary lctls and technology: the promise of open education Language Learning $\&$ Technology, 17(1), 1-6. Disponible en

http://llt.msu.edu/issues/february2013/blythcommentary.pdf

Cox, G. (2013). Researching resistance to open education resource contribution: An activity theory approach. E-Learning and Digital Media, 10(2),148-160. Disponible en

http://dx.doi.org/10.2304/elea.2013.10.2.148

Directory of Open Access Repositories (DOAR) (2012). List of repositories. Disponible en

http://www.opendoar.org/index.html

De Pablos, J. (2010). Universidad y sociedad del conocimiento. Las competencias informacionales y digitales. En: Competencias informacionales y digitales en educación superior [monográfico en línea]. Revista de universidad y sociedad del conocimiento (RUSC), r(2).doi

http://dx.doi.org/10.7238/rusc.v7i2.977

Disponible en

http://www.temoa.info/es/node/47969

Drucker, P. (2004). La sociedad post capitalista. Colombia: Editorial Norma.

European Commission (2013). Sharing knowledge: open access and preservation in Europe. Luxembourg: Publications.

http://dx.doi.org/10.2777/63410

Galina, I. \& Giménez, J. (2008). An overview of the development of open access journals and repositories in Mexico. En Proceedings ELPUB Conference en Electronic Publishing. Toronto, Canadá.

García, E. (2008). Los aprendizajes necesarios en la sociedad del conocimiento. Global, 23, 66-75. Disponible en

http://www.temoa.info/es/go/643384

García-Peñalvo, F. J. (2015). Percepciones estratégicas de la Innovación Educativa [con- 
ferencia magistral]. 45 Congreso de Investigación y Desarrollo. Disponible en

http://gredos.usal.es/jspui/han-

dle/10366/125196

García-Peñalvo, F. J., García de Figuerola, C. \& Merlo, J. A. (2010). Open knowledge: challenges and facts. Online Information Review, 34(4), 520-539.

http://dx.doi.

org/10.1108/14684521011072963

Gunasekaran, S. \& Arunachalam, S. (2014). The impact factors of open access and subscription journals across fields. Current science, 10\%(3), 380-387.

Ilkka T. (2013). Open educational resources and the transformation of education. European Journal of Education, 48(1), 58-78.

http://dx.doi.org/10.1111/ejed.12019

Lourenço, J. \& Borrell-Damian, L. (2014). Open Access to Research Publications: Looking Ahead. Bélgica: European University Association. Disponible en

http://www.eua.be

Marrero, A. (2007). La sociedad del conocimiento: una revisión teórica de un modelo de desarrollo posible para américa latina. Arxieus, 7. Disponible en:

http://www.temoa.info/es/go/113326

Melero, R., \& Hernández-San-Miguel, J. (2014). Acceso abierto a los datos de investigación, una vía hacia la colaboración científica. Revista Española de Documentación Cientifica, 37(4). doi:

http://dx.doi.org/10.3989/redc.2014.4.1154

Morgan, T. \& Carey, S. (2009). From open content to open course models: increasing access and enabling global participation in higher education. International review of research in open and distance learning, 10(5), $1-16$.

Nicolosi, G. \& Ruivenkamp, G. (2013). Re-skilling the Social Practices: Open Source and Life-Towards a Commons-Based Peer Production in Agro-biotechnology? Sci Eng Ethics, 19, 1181-1200.

http://dx.doi.org/10.1007/s11948-012-94054

Mortera, F. J. \& Escamilla, J. (2009). La iniciativa knowledge hub: Un aporte del Tecnológico de Monterrey al mundo. Revista Iberoamericana de Educación a Distancia (RIED), 12(2), 83-112.

Mortera, F. J. \& Ramírez, M. S. (2013). Conexión de repositorios educativos digitales: educonector.info. México: Lulú editorial digital. Disponible en

http://catedra.ruv.itesm.mx/han-

dle/987654321/778

en Temoa en

www.temoa.info/es/node/729370

OCDE (2007).Giving Knowledge for Free. The Emergence of Open Educational Resources. Paris, France: OECD Publishing.

RedCLARA. (2013). RedClara+Red+Ciencia. Disponible en

http//www.redclara.net/

RELPE (2011). Red Latinoamericana de Portales Educativos/Objetivos. Disponible en http://www.relpe.org

Ramírez, M. S. (2012). Academic networks and knowledge construction. Revista Españo-

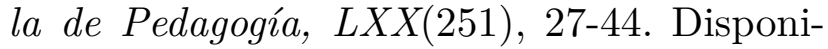
ble en

http://catedra.ruv.itesm.mx/handle/987654321/747

Ramírez, M. S. (2013). Retos y perspectivas en el movimiento educativo abierto de educación a distancia: estudio diagnóstico en un proyecto SINED. Revista de Universidad y Sociedad del Conocimiento (RUSC), 10(2), 170-186. Disponible en

http://rusc.uoc.edu/ojs/index.php/rusc/article/view/v10n2-ramirez/v10n2-ramirez-es

Ramírez, M. S. (Coord.) (2013). Competen- 
cias docentes y prácticas educativas abiertas en educación a distancia [eBook]. México: Lulú editorial digital. Disponible en http://catedra.ruv.itesm.mx/handle/987654321/745

y en Temoa en

www.temoa.info/es/node/705434

Ramírez, M. S. \& Burgos, J. V. (Coords.) (2011). Transformando ambientes de aprendizaje en educación básica con recursos educativos abiertos. Monterrey, México. Disponible en

http://catedra.ruv.itesm.mx/handle/987654321/393

Ramírez, M. S. \& Burgos, J. V. (Coords.) (2012). Movimiento educativo abierto: Acceso, colaboración y movilización de recursos educativos abiertos [eBook]. México: Lulú editorial digital. Disponible en

http://catedra.ruv.itesm.mx/han-

dle/987654321/564

y Temoa en

http://www.temoa.info/es/node/217889

Ramírez, M. S. (2014). Training strategies in team teaching to facilitate the connection of learning in MOOC courses. Edulearn14. 6th International Conference on Education and New Learning Technologies. Barcelona, España. Disponible en

http://catedra.ruv.itesm.mx/handle/987654321/841

Red Federada de Repositorios Institucionales de Publicaciones Científicas-LAReferencia (2012). LAReferencia. El proyecto. Disponible en

http://lareferencia.redclara.net/rfr/resumen

REDALYC (2012). Red de Revistas Cientificas de América Latina y el Caribe, España y Portugal. Disponible en

http://redalyc.uaemex.mx/

Rodríguez, T. M. (2011). ¿Existe Acceso Abierto en México? Presentado en el Curso Internacional de formación de Capacitadores en Escritura Científica y Acceso Abierto. Disponible en http://www.unesco.org/new/en/communication-and-information/portals-and-platforms/goap/access-by-region/latin-america-and-the-caribbean/mexico/

Rodríguez, T. M. \& Lavariega, J. C. (2011). Red Federada Latinoamericana de Repositorios Institucionales. Trabajo presentado en el Día Virtual sobre la Red Mexicana de Repositorios Institucionales, organizado por la Corporación Universitaria para Internet (CUDI). Disponible en

http://virtual.cudi.edu.mx:8080/access/content/group/bb8cda40-e844-443f-b925-7a01c6 7ac371/2011_11_10/2011_11_10

Tedesco, J. C. (2000). Educar en la sociedad del conocimiento. Buenos Aires: Fondo de Cultura Económica de Argentina.

UNESCO (2005). Towards Knowledge Societies. Paris, Francia: UNESCO.

UNESCO. (2011). Global Open Access Portal. Informativo. Disponible en http://www.unesco.org/new/en/communication-and-information/portals-and-platforms/goap/access-by-region/latin-america-and-the-caribbean/mexico/

Universidad de Guadalajara (2012). Centro de Recursos para la Enseñanza y el Aprendizaje (CREA). Disponible en http://www.udg.mx/servicios/universitarios/el-centro-recursos-la-ense-anza-y-elaprendizaje-crea

Universidad de la República (2008). Resolución No. 10 adoptada por el Consejo Directivo Central de la Universidad de la República. Uruguay. Disponible en

http://www.rau.edu.uy/docs/rescdc01.pdf

UNAM-SUAyED (2012). Recursos y Servicios Educativos. Disponible en

http://distancia.cuaed.unam.mx/recursos/

index.php

Universidad Nacional de la Plata (2012). Servicio de difusión de la creación intelectual (SEDICI) en la Universidad Nacional de la 
Plata. Disponible en

http://sedici.unlp.edu.ar/static/redirector.

php?adress=informacion/informacion
Virtual sobre la Red Mexicana de Repositorios Institucionales, organizado por la Corporación Universitaria para Internet (CUDI). Disponible en

http://virtual.cudi.edu.mx:8080/access/content/group/bb8cda40-e844-443f-b925-7a01c6 7ac371/2011_11_10/2011_11_10 